Case Report

\title{
A Case Report of High-Grade Endometrial Stromal Sarcoma: A Rare Cause of Abnormal Uterine Bleeding in a Young Woman
}

\author{
Nuntasiri Eamudomkarn (iD), Yuwadee Itarat, \\ Pilaiwan Kleebkaow, and Chumnan Kietpeerakool \\ Department of Obstetrics and Gynaecology, Faculty of Medicine, Khon Kaen University, Khon Kaen, Thailand \\ Correspondence should be addressed to Nuntasiri Eamudomkarn; pla_raa@hotmail.com
}

Received 2 October 2018; Accepted 18 November 2018; Published 28 November 2018

Academic Editor: Julio Rosa e Silva

Copyright (c) 2018 Nuntasiri Eamudomkarn et al. This is an open access article distributed under the Creative Commons Attribution License, which permits unrestricted use, distribution, and reproduction in any medium, provided the original work is properly cited.

\begin{abstract}
High-grade endometrial stromal sarcoma (HG-ESS) is a rare clinical entity, particularly among young women, and only few cases have been reported in the literature. Herein, we describe the case of a 21-year-old woman who presented with a four-month history of excessive bleeding per vagina. Endometrial curettage and cervical biopsy revealed a malignant round cell tumor suggestive of metastatic sarcoma of uterine origin. Computed tomography of the abdominopelvic region showed an enlarged uterus with diffused thickening throughout the entire endometrial cavity. Intraabdominal lymphadenopathy and ascites in the pelvic cavity were noted. The patient underwent total abdominal hysterectomy, bilateral salpingo-oophorectomy, resections of the enlarged pelvic nodes, omentectomy, and biopsy of the peritoneal nodules in the cul-de-sac. Histological examination revealed a tumor with a permeative growth pattern composed of uniformly high-grade round cells with brisk mitotic activity and extensive lymphovascular space invasion. Sections of the pelvic lymph nodes on both sides and the peritoneal nodule revealed multiple metastatic foci. Immunohistochemical studies showed positive diffuse staining for vimentin, CD 10, and cyclin D1. The pathological diagnosis was HG-ESS stage IIIC. The patient experienced rapid progression of the disease while receiving adjuvant treatment and succumbed eight months after the operation. HG-ESS is a rare cause of AUB in adolescents and young women but should be considered in the differential diagnosis.
\end{abstract}

\section{Introduction}

Abnormal uterine bleeding (AUB) is characterized as excessively heavy, prolonged, and/or frequent bleeding of uterine origin [1]. Approximately one-third of women will experience AUB during their lifetimes [2]. The most common cause of AUB in adolescents and young women is dysfunctional uterine bleeding [1]. Other possible etiologies of AUB in adolescents and young women include thyroid dysfunction, polycystic ovarian syndrome, bleeding associated with pregnancy (i.e., abortion or ectopic pregnancy), gestational trophoblastic disease, uterine leiomyoma, endometrial polyps, cervicitis, genital tract trauma, and bleeding disorders (i.e., von Willebrand disease, thrombocytopenia, or clotting factor deficiency) [1]. Cancers of the genital tract are an infrequent cause of AUB among adolescents and young women [1].

Endometrial stromal tumors (EST) are rare tumors of endometrial stromal origin and account for less than $2 \%$ of all uterine tumors [3, 4]. According to the 2014 World Health Organization (WHO) tumor classification system, EST can be classified into four categories: endometrial stromal nodule (ESN), low-grade endometrial stromal sarcoma (LG-ESS), high-grade endometrial stromal sarcoma (HG-ESS), and undifferentiated uterine sarcoma (UUS) [5].

HG-ESS is rare, particularly among young women [3]. An accumulation of reports of this rare clinical entity is, therefore, necessary to better understand its natural course. Herein, we report a case of HG-ESS in a young woman who presented with abnormal vaginal bleeding and a pelvic mass.

\section{Case Report}

A 21-year-old, G0P0 woman presented with a four-month history of excessive and prolonged bleeding per vagina, as well as a palpated mass at the lower abdomen that was rapidly increasing in size. Her past history was unremarkable. Two 


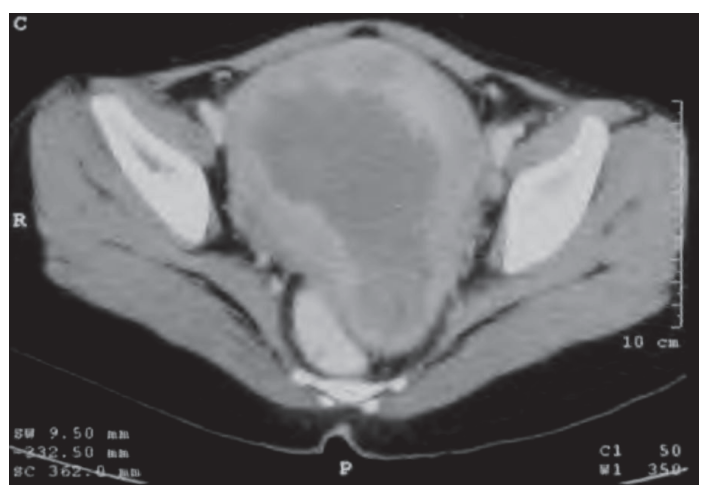

Figure 1: Computed tomography of the abdominopelvic region shows an enlarged well-defined border uterus with diffused enhancing thickening in the endometrial cavity.

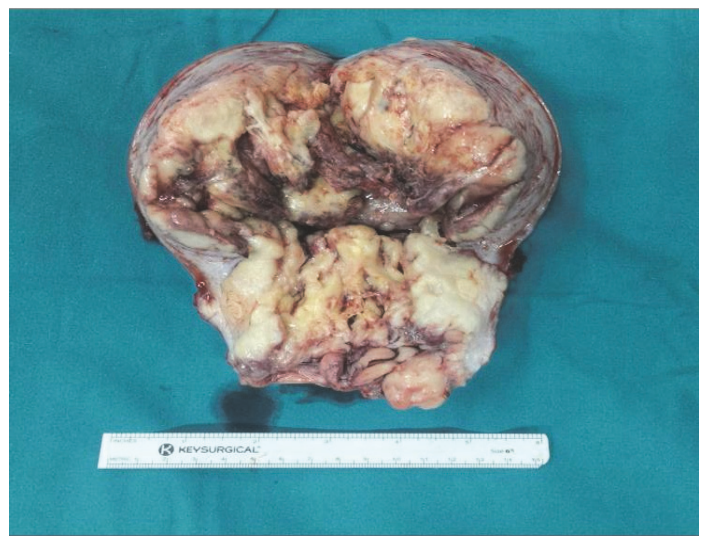

Figure 2: Gross examination of uterus reveals an enlarged uterus $13 \times 13 \times 7 \mathrm{~cm}$ with an intracavitary polypoid mass occupying the entire endometrial cavity and endocervical canal.

months before this visit, she had presented at the provincial hospital with severe anemic symptoms. She was found to have severe anemia and received a blood component transfusion. The excessive vaginal bleeding had persisted until one month prior to her presentation at our hospital. She had undergone endometrial curettage at the provincial hospital, and the pathological report indicted an atypical round cell tumor.

Upon presentation at our hospital, she was pale and found to have a midline pelvic mass. Per vaginal examination revealed a $3 \mathrm{~cm}$ exophytic mass at the posterior lip of the uterine cervix and a $14 \mathrm{~cm}$, firm uterine mass. Hematoxylineosin stained (H\&E) slides of the endometrial specimen were reviewed. The sections showed a malignant round cell tumor with scattering foci and vascular architecture mixed with benign-looking endometrial glands. Computed tomography (CT) of the abdominopelvic region showed an enlarged, well-defined uterine border with diffused enhancing thickening in the endometrial cavity involving entire uterine body and cervix (Figure 1). The overall uterine size was $15.0 \times 11.6 \times 10.5 \mathrm{~cm}$. Intra-abdominal lymphadenopathy and ascites in the pelvic cavity were noted. The liver, gallbladder, pancreas, spleen, bilateral kidneys, and bilateral adrenal glands appeared normal.
A biopsy of the cervical mass was performed to obtain tissue for further study of immunohistochemical (IHC) markers. The pathological examination showed a malignant small round cell tumor suggestive of metastatic sarcoma of uterine origin. Immunohistochemical studies showed negative staining for multi-cytokeratin (AE1/AE3), S-100 protein, CD 10, cyclin D1, caldesmon, myogenin, and desmin. The patient's tumor exhibited focal positive staining for smooth muscle actin. The preoperative differential diagnoses were HG-ESS and UUS.

At laparotomy, the uterus and obturator lymph nodes on both sides were enlarged. There were multiple nodules in the cul-de-sac. Neither the adnexae nor the omentum appeared remarkable. The surgical procedures included total abdominal hysterectomy, bilateral salpingo-oophorectomy, resections of enlarged pelvic nodes, omentectomy, and biopsy of peritoneal nodules in the cul-de-sac.

Macroscopically, the uterus weighed 714.67 grams and measured $13 \times 13 \times 7 \mathrm{~cm}$ with an intracavitary polypoid mass that occupied the entire endometrial cavity (Figure 2). The tumor had invaded the serosa of the uterus and ectocervix. Sectioning revealed a yellow cut surface with focal areas of hemorrhage. Histologically, the tumor exhibited a permeative 


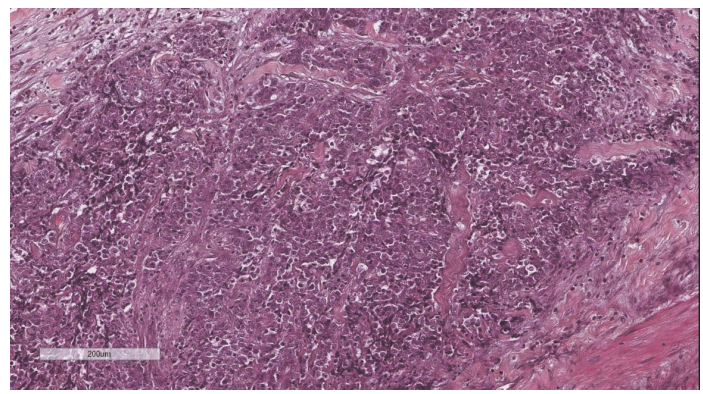

Figure 3: Microscopic examination reveals a small round cell tumor with high mitotic activity arranged in tight nests separated by a delicate capillary network (H\&E staining; 20x).

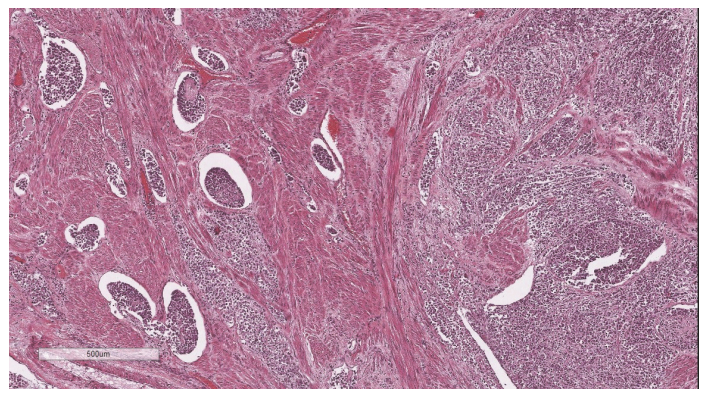

FIGURE 4: Microscopic examination reveals the presence of an extensive lymphovascular space invasion in the myometrium (H\&E staining; $4 \mathrm{x})$.

growth pattern and was composed of uniformly high-grade round cells with brisk mitotic activity arranged in tight nests separated by a delicate capillary network (Figure 3 ). The tumor had invaded the uterine serosa, and there was extensive lymphovascular space invasion (LVSI) (Figure 4). Sections of the pelvic lymph nodes on both sides and peritoneal nodule revealed multiple metastatic foci. The omentum, both ovaries, and both fallopian tubes were histologically unremarkable. Additional IHC studies revealed positive diffuse staining for vimentin, CD 10, and cyclin D1. The tumor stained negative for desmin, estrogen receptors (ER), and progesterone receptors (PR). A diagnosis of HG-ESS stage IIIC was made based on these pathological findings.

The postoperative clinical course was uneventful. The patient was started on adjuvant chemotherapy consisting of Adriamycin $\left(50 \mathrm{mg} / \mathrm{m}^{2}\right)$ and Ifosfamide $\left(5 \mathrm{~g} / \mathrm{m}^{2}\right)$ given every three weeks. After receiving four courses of this chemotherapy regimen, the patient refused further adjuvant chemotherapy due to her inability to tolerate the side-effects. Pelvic radiation was then administered thereafter. Despite undergoing radiation treatment, the patient experienced rapid disease progression and succumbed eight months after operation.

\section{Discussion}

Abnormal uterine bleeding is a common reason for adolescents and young women to present at healthcare facilities [2]. There are various causes of AUB in these patients, and cancer of the genital tract is relatively rare [1]. Possible genital tract cancers that cause AUB in adolescents and young women include endometrial cancer, vaginal rhabdomyosarcoma, and cervical adenocarcinoma [6-8]. In this report, we describe a case of HG-ESS in a 21-year-old woman who presented with abnormal vaginal bleeding and a pelvic mass.

High-grade endometrial stromal sarcoma is rare, constituting of less than $1 \%$ of uterine malignancies and less than $10 \%$ of uterine sarcomas $[3,4]$. The common presenting symptoms of HG-ESS are abnormal vaginal bleeding, palpable masses, and pelvic pain [3]. The clinical presentations in our patient are generally consistent with those that have previously been reported except the patient's age. The average ages at HG-ESS diagnosis reported in the literature range from 40 years to 55 years [3]. The exceptional finding in our report, thus, is HG-ESS being diagnosed in a young patient.

Endometrial stromal sarcoma is a genetically heterogenous group of uterine sarcomas [9-11]. High-grade endometrial stromal sarcoma typically harbors $\mathrm{t}(10 ; 17)(\mathrm{q} 22 ; \mathrm{p} 13)$ resulting in YWHAE-NUTM2A/B (previously known as YWHAE- FAM22) genetic fusion [5]. YWHAE-rearranged HG-ESS represents a molecularly and prognostically distinct type of ESS, which is associated with an aggressive natural course $[10,11]$. The unique clinical behavior and treatment responses of this subset of ESS therefore merit pathologic evaluation to confirm the presence of the $t(10 ; 17)(q 22 ; p 13)$ translocation in cases of morphologically suspected HG-ESS [10]. Cyclin D1 has been proposed as a sensitive and specific diagnostic immunomarker for YWHAE-FAM22 ESS [9]. In a recent case series, the high-grade round cell component of all 12 YWHAE-FAM22 ESS exhibited diffuse moderate to 
strong nuclear cyclin D1 staining, and this diffuse positivity has not been seen in patients who harbored other genetic rearrangements [9]. The authors postulate the utility of cyclin D1 staining to indicate a provisional diagnosis of YWHAE-FAM22 ESS when encountering a uterine tumor in which the differential diagnosis of a histologically HG-ESS is considered [9]. In our patient, there was diffuse positive cyclin D1 staining, possibly indicating a provisional diagnosis of YWHAE-rearranged HG-ESS.

High-grade endometrial stromal sarcoma is also a group of tumors with heterogenous morphologic features [12]. Sciallis et al. [12] classified HG-ESS into three subgroups based on morphology: (I) tumors with a component that is similar to LG-ESS and transitions abruptly into a highergrade component; (II) tumors composed exclusively of highgrade round cells with uniform nuclear features but with a permeative pattern of infiltration; and (III) tumors similar to the second group but with cytomorphology featuring enlarged round to ovoid cells with smooth nuclear membranes and distinct chromatin clearing but lacking prominent nucleoli. Lymphovascular space invasion is commonly noted in category III tumors [12]. In addition, the authors observed that most of the tumors in category III tested positive for YWHAE rearrangement [12]. Thus, cytomorphologic features on routine hematoxylin and eosin-stained sections may be helpful in classifying tumors with different molecular genetic changes.

According to the National Cancer Database, which is a nationwide, facility-based, comprehensive database established by the American Cancer Society and Commission on Cancer of the American College of Surgeons, survival in patients with HG-ESS remains poor [13]. The median overall survival was 19.9 months (95\% CI, 17.1-22.1 months), and the five-year overall survival was only $32.6 \%$ (95\% CI; $30.1-$ $35.3 \%)$. Prognostic factors negatively associated with survival include patients' age, tumor size, omission of lymphadenectomy, pathologically positive resection margins, and distant or nodal metastasis [13]. Some of these poor prognostic factors were noted in our patient including large tumor size and metastases to the peritoneum and pelvic lymph nodes.

It is extremely rare for HG-ESS to be diagnosed in adolescents and young women, and only a few cases have been reported in the literature [14-16]. The prognosis of HGESS among this subset of women is generally poor due to the disease presenting at a later stage. Patients in some of the reported cases have succumbed to the extensive metastases of the disease in a relatively short time period after surgery $[14,15]$. In our patient, the tumor rapidly progressed during adjuvant treatment and the patient passed away at eight months after the operation. The strength of this report is that the diagnosis of HG-ESS was confirmed by a panel of IHC staining. However, the definite molecular genetic change in our case was unknown.

\section{Conclusion}

We describe a case of HG-ESS stage IIIC diagnosed in a 21-year-old woman who presented with abnormal vaginal bleeding and a pelvic mass. Our report confirms the relatively poor prognosis of this subtype of ESS. Our patient experienced rapid disease progression during adjuvant treatment and passed away at eight months after surgery. This report highlights that HG-ESS is a rare cause of AUB in adolescents and young women but should be considered in the differential diagnosis.

\section{Data Availability}

The data used to support the findings of this study are available from the corresponding author upon request.

\section{Consent}

Written informed consent was obtained from the patient for publication of this case report.

\section{Conflicts of Interest}

The authors declare that there are no conflicts of interest regarding the publication of this paper.

\section{Acknowledgments}

The authors are grateful to Mr. Dylan Southard for his assistance in editing the manuscript.

\section{References}

[1] S. Elmaoğulları and Z. Aycan, "Abnormal Uterine Bleeding In Adolescents," Journal of Clinical Research in Pediatric Endocrinology, vol. 10, no. 3, pp. 191-197, 2018.

[2] L. J. Benjamins, "Practice Guideline: Evaluation and Management of Abnormal Vaginal Bleeding in Adolescents," Journal of Pediatric Health Care, vol. 23, no. 3, pp. 189-193, 2009.

[3] J. A. Rauh-Hain and M. G. del Carmen, "Endometrial stromal sarcoma: a systematic review," Obstetrics \& Gynecology, vol. 122, no. 3, pp. 676-683, 2013.

[4] C. M. J. Conklin and T. A. Longacre, "Endometrial stromal tumors: The new who classification," Advances in Anatomic Pathology, vol. 21, no. 6, pp. 383-393, 2014.

[5] R. H. Ali and M. Rouzbahman, "Endometrial stromal tumours revisited: An update based on the 2014 WHO classification," Journal of Clinical Pathology, vol. 68, no. 5, pp. 325-332, 2015.

[6] J. Hanprasertpong, S. Sakolprakraikij, and A. Geater, "Endometrial cancer in Thai women aged 45 years or younger," Asian Pacific Journal of Cancer Prevention, vol. 9, no. 1, pp. 58-62, 2008.

[7] M. Baykara, M. Benekli, O. Erdem et al., "Clear cell adenocarcinoma of the uterine cervix: A case report and review of the literature," Journal of Pediatric Hematology/Oncology, vol. 36, no. 2, pp. e131-e133, 2014.

[8] A. Sachedina, K. Chan, D. MacGregor, M. Campbell, and S. R. Grover, "More than Grapes and Bleeding: An Updated Look at Pelvic Rhabdomyosarcoma in Young Women," Journal of Pediatric \& Adolescent Gynecology, vol. 31, no. 5, pp. 522-525, 2018.

[9] C.-H. Lee, R. H. Ali, M. Rouzbahman et al., "Cyclin D1 as a diagnostic immunomarker for endometrial stromal sarcoma 
with YWHAE-FAM22 rearrangement," The American Journal of Surgical Pathology, vol. 36, no. 10, pp. 1562-1570, 2012.

[10] C.-H. Lee, A. Mariño-Enriquez, W. Ou et al., "The clinicopathologic features of YWHAE-FAM22 endometrial stromal sarcomas: A histologically high-grade and clinically aggressive tumor," The American Journal of Surgical Pathology, vol. 36, no. 5, pp. 641-653, 2012.

[11] M. L. Hemming, A. J. Wagner, M. R. Nucci et al., "YWHAErearranged high-grade endometrial stromal sarcoma: Twocenter case series and response to chemotherapy," Gynecologic Oncology, vol. 145, no. 3, pp. 531-535, 2017.

[12] A. P. Sciallis, P. P. Bedroske, J. K. Schoolmeester et al., "Highgrade endometrial stromal sarcomas: A clinicopathologic study of a group of tumors with heterogenous morphologic and genetic features," The American Journal of Surgical Pathology, vol. 38, no. 9, pp. 1161-1172, 2014.

[13] B.-L. L. Seagle, A. Shilpi, S. Buchanan, C. Goodman, and S. Shahabi, "Low-grade and high-grade endometrial stromal sarcoma: A National Cancer Database study," Gynecologic Oncology, vol. 146, no. 2, pp. 254-262, 2017.

[14] S. Michalas, O. Creatsas, E. Deligeoroglou, and S. Markaki, "High-grade endometrial stromal sarcoma in a 16-year-old girl," Gynecologic Oncology, vol. 54, no. 1, pp. 95-98, 1994.

[15] K. C. Wang, D. C. Liang, T. H. Su, F. Y. Hung, and Y. C. Yang, "High-grade endometrial stromal sarcoma in a 10-year-old girl: case report," Changgeng Yi Xue Za Zhi, vol. 21, no. 3, p. 312, 1998.

[16] C. Emmert, F. Dietrich, W. D. Wecke, and H.-H. Riedel, "High-grade endometrial stromal sarcoma (HG-ESS) in an adolescent girl with benign mediastinal Schwannoma," Journal of Obstetrics \& Gynaecology, vol. 19, no. 2, pp. 210-212, 1999. 


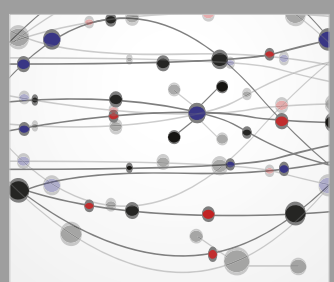

The Scientific World Journal
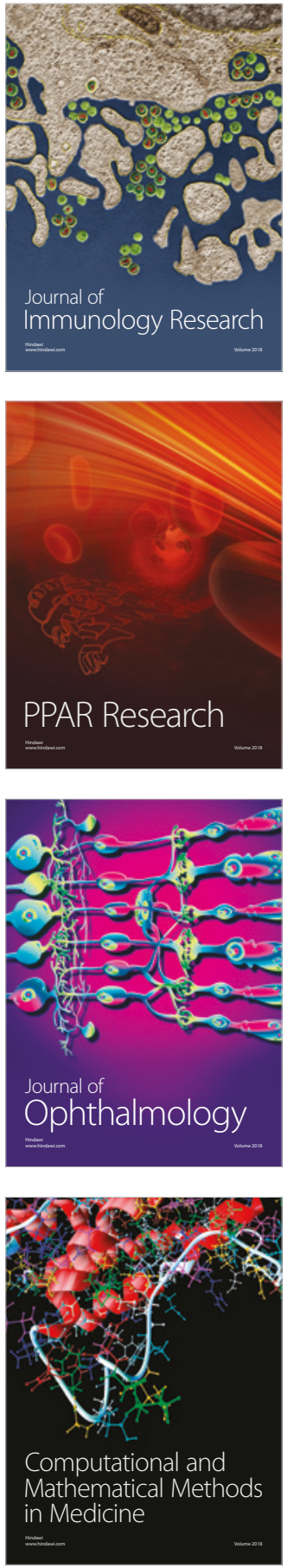

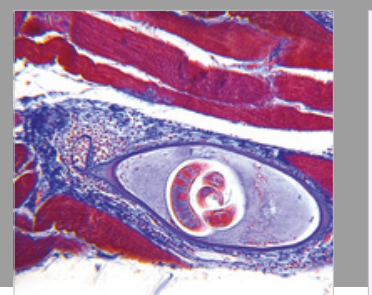

Gastroenterology Research and Practice

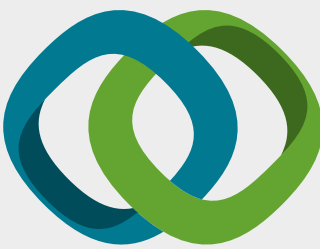

\section{Hindawi}

Submit your manuscripts at

www.hindawi.com
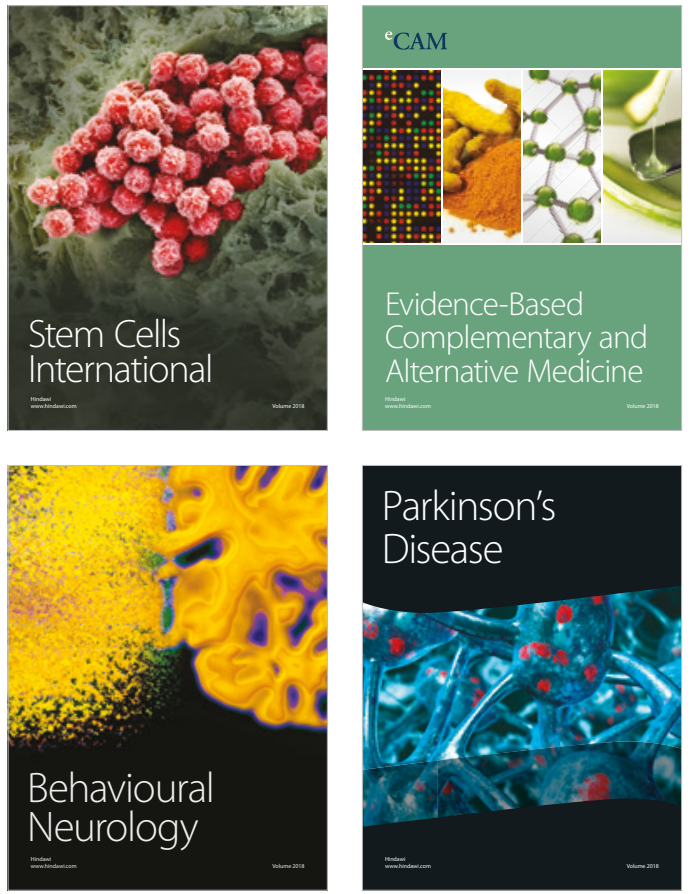

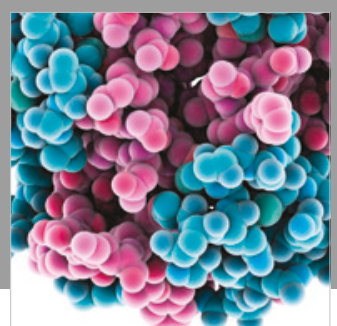

ournal of

Diabetes Research

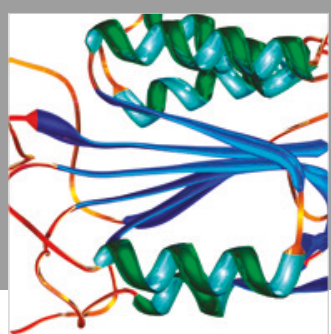

Disease Markers
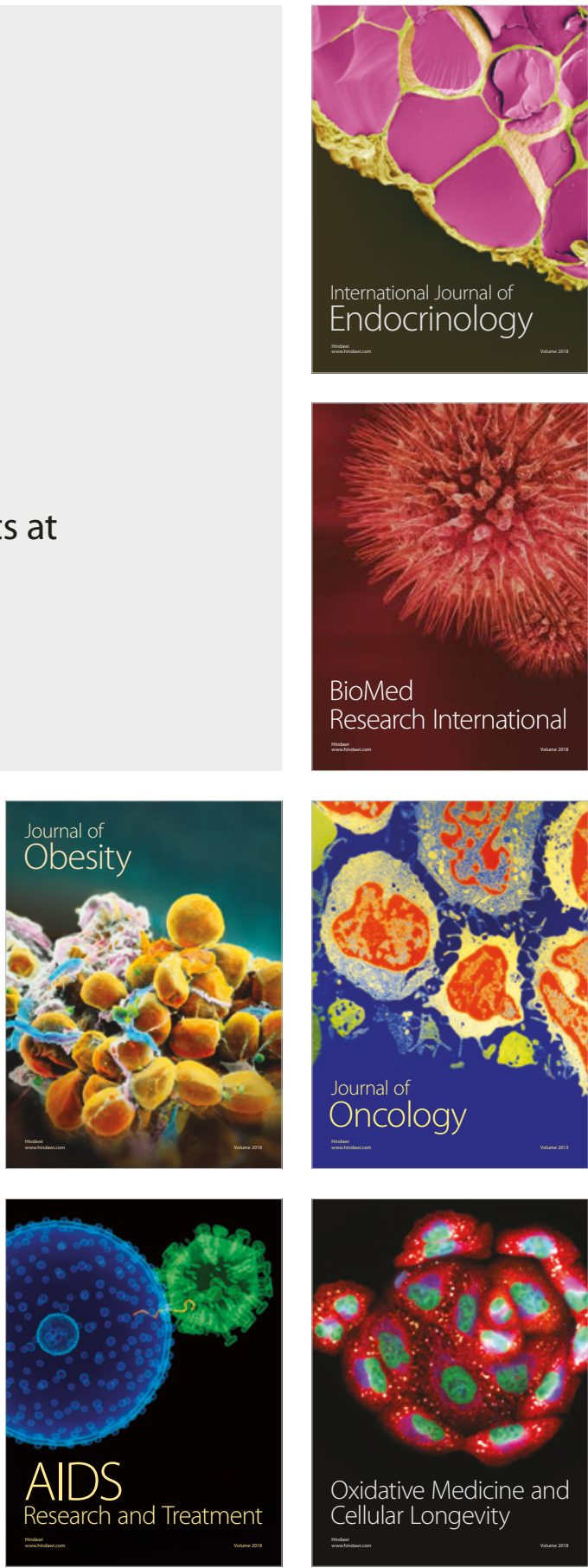